\title{
Brownian dynamics simulation of linear polymers under elongational flow: Bead-rod model with hydrodynamic interactions
}

\section{Citation for published version (APA):}

Neelov, I. M., Lyulin, A. V., Adolf, D. B., \& Davies, G. R. (2002). Brownian dynamics simulation of linear polymers under elongational flow: Bead-rod model with hydrodynamic interactions. Journal of Chemical Physics, 117(8), 4030-4041. https://doi.org/10.1063/1.1493187

DOI:

10.1063/1.1493187

Document status and date:

Published: 01/01/2002

\section{Document Version:}

Publisher's PDF, also known as Version of Record (includes final page, issue and volume numbers)

\section{Please check the document version of this publication:}

- A submitted manuscript is the version of the article upon submission and before peer-review. There can be important differences between the submitted version and the official published version of record. People interested in the research are advised to contact the author for the final version of the publication, or visit the $\mathrm{DOI}$ to the publisher's website.

- The final author version and the galley proof are versions of the publication after peer review.

- The final published version features the final layout of the paper including the volume, issue and page numbers.

Link to publication

\footnotetext{
General rights

- You may freely distribute the URL identifying the publication in the public portal. follow below link for the End User Agreement:

www.tue.nl/taverne

\section{Take down policy}

If you believe that this document breaches copyright please contact us at:

openaccess@tue.nl

providing details and we will investigate your claim.
}

Copyright and moral rights for the publications made accessible in the public portal are retained by the authors and/or other copyright owners and it is a condition of accessing publications that users recognise and abide by the legal requirements associated with these rights.

- Users may download and print one copy of any publication from the public portal for the purpose of private study or research.

- You may not further distribute the material or use it for any profit-making activity or commercial gain

If the publication is distributed under the terms of Article 25fa of the Dutch Copyright Act, indicated by the "Taverne" license above, please 


\title{
Brownian dynamics simulation of linear polymers under elongational flow: Bead-rod model with hydrodynamic interactions
}

\author{
Igor M. Neelov and David B. Adolf \\ Department of Physics and Astronomy, University of Leeds, Leeds LS2 9JT, United Kingdom \\ Alexey V. Lyulin \\ Eindhoven University of Technology and Dutch Polymer Institute, P.O. Box 513, 5600 MB, Eindhoven, \\ The Netherlands \\ Geoffrey R. Davies \\ Department of Physics and Astronomy, University of Leeds, Leeds LS2 9JT, United Kingdom
}

(Received 1 November 2001; accepted 21 May 2002)

\begin{abstract}
Brownian dynamics (BD) simulations of a linear freely jointed bead-rod polymer chain with excluded volume (EV) interaction have been performed under elongational flow with and without the use of fluctuating hydrodynamic interactions (HI). The dependence of the chain size, shape and intrinsic elongational viscosity on the elongational rate $\dot{\varepsilon}$ are reported. A sharp coil-stretch transition is observed when $\dot{\varepsilon}$ exceeds a critical value, $\dot{\varepsilon}_{c}$. The inclusion of the HI leads to a shift in the coil-stretch transition to higher flow values. Chain deformation due to elongational flow is observed to first consist of the alignment of the chain with the direction of flow without significant chain extension followed by additional alignment of the bond vectors with the flow direction and chain extension as flow rate is increased further. The distribution function for the chain's radius of gyration becomes significantly broader within the transition region which implies an increase in fluctuations in the chain size in this region. The structure factors parallel and perpendicular to the flow direction illustrate different elongational rate dependencies. At high rates, the structure factor in the direction of the flow exhibits an oscillating dependence which corresponds to the theoretically predicted shape for a rigid-rod model. The mean squared orientation of each bond within the chain with respect to the flow direction as function of bond number is nearly parabolic in shape with the highest degree of orientation found within the chain's interior. The dependence of the critical elongational rate, $\dot{\varepsilon}_{c}$, on the chain length, $N$, is observed to be $\dot{\varepsilon}_{c} \sim N^{-1.96}$ when hydrodynamic interactions are not employed and $\dot{\varepsilon}_{c} \sim N^{-1.55}$ when they are invoked. These scaling exponents agree well with those obtained in previous BD simulations of bead-FENE (i.e., finitely extensible nonlinear elastic) spring chains as well as with the theoretical predictions of $\dot{\varepsilon}_{c} \sim N^{-2}$ and $\dot{\varepsilon}_{c}$ $\sim N^{-1.5}$ without and with hydrodynamic interactions based on the Rouse and Zimm models, respectively. (C) 2002 American Institute of Physics. [DOI: 10.1063/1.1493187]
\end{abstract}

\section{INTRODUCTION}

The response of a dilute solution of long polymer chains to elongational flow has been of interest to the polymer physics community for several decades. ${ }^{1-13}$ This issue is relevant to important applications such as turbulent drag reduction and enhanced oil recovery. Frank et al. ${ }^{1}$ used an opposing flow apparatus to first observe the currently well-known sharp transition of a flexible macromolecule between a coiled state and a stretched state (i.e., the coil-stretch transition) under the influence of an elongational flow. ${ }^{1,2}$ This phenomenon occurs when the elongational rate, $\dot{\varepsilon}$, exceeds a critical value, $\dot{\varepsilon}_{c}$, and has been observed for a variety of chemically distinct polymers. ${ }^{2-7}$ Direct visualization of a conformation of a single DNA molecule in an elongational flow are presented in the works of Perkins and co-workers. ${ }^{2,7}$ A theoretical investigation into the behavior of a linear polymer chain in dilute solution under elongational flow was first performed by de Gennes. ${ }^{8}$ Within this effort, the chain is represented as a dumbbell with a conformationally dependent elasticity and friction and invokes the Peterlin-de
Gennes approximation $(\mathrm{P}-\mathrm{dG}) .{ }^{8,9}$ It was shown that the coil-stretch transition may be continuous ("second order") or discontinuous ("first order"). Similar ideas have been presented by Hinch. ${ }^{10}$ A three-dimensional dumbbell model with a conformational dependent friction coefficient was used by Brestkin ${ }^{11}$ where the $\mathrm{P}-\mathrm{dG}$ approximation was not invoked. Despite this, a discontinuous coil-stretch transition was obtained.

Magda et al. ${ }^{12}$ modified Fixman's technique ${ }^{13}$ and applied it to a bead-spring Zimm model with up to 500 beads and preaveraged hydrodynamic interactions that depend on deformation rate. It was revealed that the incorporation of these conformational-dependent hydrodynamic interactions shifted the onset of the coil-stretch transition relative to that predicted by Zimm. Bead-FENE (i.e., finitely extensible nonlinear elastic) spring chains were considered within the theoretical efforts of Wiest et al. ${ }^{14}$ Chains of length 7, 10, and 20 beads were employed. It was observed that the coilstretch transition for this model is gradual and that springs within the middle of the chain were slightly more extended 
at intermediate flow rates. Four different stages of extension were identified as the equilibrium coil, the deformed coil, the stretched spring, and the unfolded chain. Further detailed information on theoretical and experimental efforts dealing with linear polymer chains in elongational flow is found within Ref. 2.

Within the last three decades, molecular dynamics (MD) and Brownian dynamics (BD) computer simulations methods ${ }^{15-41}$ have emerged as powerful tools not only for the confirmation of theoretical predictions but equally, if not more so, for the unique insight that they offer in their own right. The first $\mathrm{MD}^{15-17}$ and $\mathrm{BD}^{18,20-26}$ efforts addressed the behavior of both unperturbed linear polymer chains and chains in various external fields using both elastic and rigid bond chain models. The BD investigations usually did not take hydrodynamic interactions (HI) into account. Acierno et al. ${ }^{18}$ and Rallison and Hinch ${ }^{22}$ simulated bead-rod chains without $\mathrm{HI}$ in uniaxial flow and observed that the unfolding of back loops leads to large viscous stresses. Larson developed a simple dynamic kink model ${ }^{42}$ to describe this backloop unfolding and obtained that the viscous stress scaled with the third power of the end-to-end distance, $\mathrm{Hinch}^{23}$ used a three-dimensional model of a bead-rod chain without $\mathrm{HI}$ in addition to a one dimensional model of kink dynamics to describe chain uncoiling in elongational flow. It was observed that at large extensions the elongational flow rate dependence of the end-to-end distance were similar for both models.

$\mathrm{Liu}^{24}$ proposed an algorithm to simulate the motion of a freely jointed bead-rod chain with constant hydrodynamic interactions in steady state shear and elongational flow. For elongational flow, he addressed the elongational rate dependence of the steady state elongational viscosity and the mean squared end-to-end distance for chains of 10 and 20 beads. It was observed that for longer chains the coil-stretch transition was sharper and occurred at smaller elongational rates. The rheological and optical behavior of a Kramers bead-rod chain without $\mathrm{HI}$ in dilute solution under uniaxial extensional flow were studied by Doyle et al. ${ }^{25}$ They used the algorithm suggested by Liu and showed that the Brownian stress dominates in steady state uniaxial extensional flow at small flow rates and that the stress-optical law is valid within this region. Larson et al. ${ }^{26}$ used the BD method to investigate the steady state extensional behavior of a beadspring chain model without HI but with a "wormlike" elasticity for each spring. Snapshots of this wormlike polymer chain were compared with snapshots of a single DNA molecule within elongational flow obtained by Perkins with co-authors ${ }^{2,7}$ within the laboratory. The dependence of chain extension on elongational rate was also intercompared. Significant "molecular individualism" 43 was observed arising from a dependence of the onset and the shape of the coilstretch transition on the initial configuration of the molecule.

Darinskii and co-workers ${ }^{27,28}$ employed Brownian dynamics computer simulations to study the coiling and uncoiling of a polymer chain modeled as a dumbbell with conformational dependent parameters by Brownian dynamics. They calculated the effective potential ${ }^{8}$ due to de Gennes for this model and investigated the dependence of the average end- to-end distance on elongational rate. At elongational flow rates near a critical value, they found that the distribution function of the end-to-end distance is bimodal reflecting the presence of a mixture of coiled and stretched chains. The same dumbbell model was used later ${ }^{29}$ for the BD simulation of a polymer chain in oscillatory elongational planar flow between two linear arrays of rotating cylinders.

Some of the first computational investigations into the elongational rate dependence of the chain size and the intrinsic elongational viscosity of bead-spring polymer chains with rigorous inclusion of $\mathrm{HI}$ and excluded volume (EV) have been carried out by de la Torre and co-workers ${ }^{30-35}$ over the last 10 years. These studies employed a variety of different soft potentials to constrain the bond lengths (i.e., Rouse, Morse, FENE springs) and reported the dependence of the chain end-to-end distance on elongational rate. The transition between coiled and stretched states defined to occur at $\dot{\varepsilon}_{c}$ was studied as a function of chain length, $N$. It is reported ${ }^{35}$ that for FENE chain $\dot{\varepsilon}_{c} \sim N^{-1.55}$ both for theta and good solvent conditions when HI were taken into account and $\dot{\varepsilon}_{c} \sim N^{-2.00}$ without HI.

The steady state and transient rheological behavior of bead-FENE spring chains with and without the inclusion of $\mathrm{EV}$ and $\mathrm{HI}$ in extensional flow has also been studied by Fetsko and Cummings. ${ }^{36}$ The parameter setting the maximum extent of extension of the FENE springs was observed to have a pronounced effect on the simulated results by controlling the dependence of the elongational viscosity on elongational rate. For example, for the situation with EV and without $\mathrm{HI}$, the elongational viscosity increased with elongational rate for a chain with soft FENE bonds but decreased with elongational rate for chains with stiff FENE bonds. Clarification of any confusion generated by this behavior is available only through similar elongational flow simulations using chain models with rigid bond lengths both with and without HI.

To the authors' knowledge, the only simulation of a bead-rod model of a linear polymer chain in elongational flow and in the presence of $\mathrm{HI}$ are the two recent efforts of Agarwal et al. ${ }^{37}$ They used one chain length of $N=100$ and EV were not invoked. The SHAKE-HI algorithm ${ }^{41}$ was employed which has been criticized from a theoretical viewpoint by Öttinger. ${ }^{44,45}$ The behavior of the chain within the flow was characterized in the first paper solely by the change in the end-to-end distance which was observed to undergo a sharp coil-stretch transition above a critical rate. Incorporation of HI displaced this value of $\dot{\varepsilon}_{c}$ to higher flow values. The intrinsic elongational viscosity was calculated only in the absence of HI. In the second paper, ${ }^{37}$ the behavior of 10 individual chains with different initial conformations was calculated with and without HI. The initial conformation of the chain was observed to influence greatly the chain extension process in agreement with the earlier experimental efforts of Perkins and co-workers ${ }^{7}$ and the simulation work of Larson and co-workers. ${ }^{26}$

In the present paper, Brownian dynamics simulations of freely jointed bead-rod chains of different length in steadystate elongational flow are performed for the first time in the presence of both EV and fluctuating HI. A similar chain 
without HI is studied for comparison. The simulations are analyzed in terms of

(1) the average size and the shape of the chain as a whole;

(2) the structure factor of the deformed chain at different elongational rates;

(3) the local orientation of each chain monomer along the chain;

(4) the birefringence and stress-optical relationship under the influence of elongational flow;

(5) the dependence of the intrinsic elongational viscosity on the elongational flow rate; and

(6) the dependence of the critical elongational rates, $\dot{\varepsilon}_{c}$, on chain length.

This paper also represents the first paper in a series of articles on the behavior of chains with different architectures in elongational flow. It complements an earlier investigation ${ }^{38}$ of linear polymers, dendrimers and hyperbranched polymers employing a bead-rod model with EV and HI within a shear flow. The use of rigid connectors affords better insight into structural and dynamic details occurring on a more local level where bonds are not deformed as much at high elongational rates as they would be within a FENE chain model. In Sec. II, the model of the polymer chain is introduced and the details of the simulation algorithm are explained. The simulated behavior of the chain under elongational flow is reported in Sec. III. A general summary is presented in Sec. IV.

\section{MODEL AND ALGORITHM DETAILS}

The chain is composed of $N$ identical centers of viscous friction $\zeta$ linked by $N_{\mathrm{b}}=N-1$ rigid rods of length $l$. Within a Brownian dynamics simulation, the motion of the simulated particles is dictated by the Langevin equation in the high friction limit where the inertial term is neglected. Brownian dynamics algorithms to simulate the motion of a bead-rod polymer chains with HI have been proposed by Fixman, ${ }^{39}$ Ermak and McCammon, ${ }^{40}$ Öttinger ${ }^{44,45}$ and others. This investigation employs the approach used in our previous simulations ${ }^{38}$ of linear polymer chains, dendrimers and hyperbranched polymers in shear flow. For this study, two modifications were made to the algorithm. First, all calculations are performed within the frame of the center of mass of the polymer chain. Second, the calculation of the constraint forces due to the rigid bonds (see below) is performed in a fashion similar to Öttinger. ${ }^{44,45}$

The equation of motion has the form

$$
\begin{gathered}
\vec{r}_{i}=\vec{r}_{i}^{0}+\frac{\Delta t}{k_{B} T} \sum_{j} \mathbf{D}_{i j}^{0} \cdot \vec{F}_{j}^{0}+\vec{\nu}_{i}^{0} \cdot \Delta t+\vec{\Phi}_{i}^{0}(\Delta t), \\
i=1, \ldots, N,
\end{gathered}
$$

where $\vec{r}_{i}^{0}$ and $\vec{r}_{i}$ are the position vectors for bead $i$ before and after an unconstrained timestep $\Delta t, k_{B} T$ is the Boltzmann factor, $\mathbf{D}_{i j}^{0}$ is the diffusion tensor, and $\vec{\nu}_{i}^{0}$ is the velocity of the solvent at the position of bead $i$. For steady uniaxial elongational flow with an elongational rate of $\dot{\varepsilon}, \nu_{i, x}^{0}=x_{i}^{0} \dot{\varepsilon}, \nu_{i, y}^{0}=$ $-0.5 y_{i}^{0} \dot{\varepsilon}, \nu_{i, z}^{0}=-0.5 z_{i}^{0} \dot{\varepsilon}$, where the direction of flow lies along the $x$ axis. $\nu_{i x}^{0}, \nu_{i y}^{0}$, and $\nu_{i z}^{0}$ are the components of the solvent velocity at the position $x_{i}^{0}, y_{i}^{0}$, and $z_{i}^{0}$ of the $i$ th bead. The solvent within these simulations is represented as a structureless continuum with chain-solvent collisions mimicked by the vector $\vec{\Phi}_{i}^{0}$ which has a variance-covariance matrix given by

$$
\left\langle\vec{\Phi}_{i}^{0}(\Delta t) \vec{\Phi}_{j}^{0}(\Delta t)\right\rangle=2 \Delta t \mathbf{D}_{i j}^{0} .
$$

For the case when HI are neglected, one has

$$
\begin{aligned}
& D_{i i}^{(\alpha \beta) 0}=\left(k_{B} T / \zeta\right) \delta_{\alpha \beta}, \quad i=1, \ldots, N, \\
& D_{i j}^{(\alpha \beta) 0}=0, \quad \text { for } i \neq j=1, \ldots, N,
\end{aligned}
$$

where $\delta_{\alpha \beta}$ is a unit matrix $(\alpha, \beta=x, y, z)$. A constraint force, $\vec{F}_{j}$, acting on a bead $j$ arises from neighboring beads along the chain according to

$$
\vec{F}_{j}=-\sum_{k=1}^{N-1} \lambda_{k}\left(\frac{\partial \sigma_{k}}{\partial \vec{r}_{j}}\right)_{\mathbf{r}},
$$

where $\sigma_{k}$ is an equation of rigid constraint for the $k$ th bond,

$$
\sigma_{k}=\left(\vec{r}_{k+1}-\vec{r}_{k}\right)^{2}-l^{2}, \quad k=1, \ldots, N-1
$$

and $\lambda_{k}$ is a corresponding Lagrange multiplier. The bead positions and the diffusion tensor employed in $\mathrm{SHAKE}^{15}$ and SHAKE- $\mathrm{HI}^{41}$ are based on trajectory points of the previous time step. Within this study, the bead positions and the diffusion tensor employed are based on trajectory points midway between the previous time step and the current unconstrained step which is consistent with the approach suggested by Öttinger ${ }^{44,45}$ in addition to the recent efforts of Petera and Muthukumar $^{46}$ which employed the Öttinger algorithm within BD simulations of a freely jointed bead-rod chain with $\mathrm{EV}$ and $\mathrm{HI}$ in shear flow.

A further contribution to the force on a given bead $j$ arises from the Lennard-Jones potential, $U_{\mathrm{LJ}}$, imposed between beads $i$ and $j$ separated by more than one bond where

$$
U_{\mathrm{LJ}}=\sum_{|i-j| \geqslant 4} 4 \varepsilon_{0}\left(\left(\frac{\sigma}{r_{i j}}\right)^{12}-\left(\frac{\sigma}{r_{i j}}\right)^{6}\right)+U^{\text {cutoff }} .
$$

The parameters $\sigma=0.8 l$ and $\varepsilon_{0}=0.3 k_{B} T$ are used as proposed by Rey et al. ${ }^{19}$ to reproduce correctly the molecularweight dependence of the mean squared end-to-end distance, $\left\langle R^{2}\right\rangle$, on chain length for a linear chain in a $\theta$ solution. A cutoff of $2.5 \sigma$ is also invoked and $U^{\text {cutoff }}-U(2.5 \sigma)$. HI are represented rigorously by means of the Rotne-PragerYamakawa interaction tensor. ${ }^{47,48}$ In this case diagonal elements of the diffusion tensor $\mathbf{D}_{i i}$ are given by Eq. (3a) and for nonoverlapping beads one has

$$
\begin{aligned}
D_{i j}^{\alpha \beta}= & h^{*}(\pi / 3)^{1 / 2}\left(3 k_{B} T / 4 \zeta\right)\left(l / R_{i j}\right) \\
& \times\left[\left(\delta_{\alpha \beta}+\frac{R_{i j}^{\alpha} R_{i j}^{\beta}}{R_{i j}^{2}}\right)+\frac{2 a^{2}}{3 R_{i j}^{2}}\left(\delta_{\alpha \beta}-\frac{3 R_{i j}^{a} R_{i j}^{\beta}}{R_{i j}^{2}}\right)\right],
\end{aligned}
$$

where $R_{i j}$ is the separation between beads $i$ and $j$ each with hydrodynamic radius $a$ and $\zeta$ is the friction coefficient. The strength of the HI effects is represented by the parameter $h^{*}=(3 / \pi)^{1 / 2} a / l$. A value of $h^{*}=0.25$ is used which corresponds to the hydrodynamic radius $a=0.257 l$. If the beads are allowed to overlap $\left(R_{i j}<2 a\right)$ this tensor is 


$$
D_{i j}^{\alpha \beta}=\left(k_{B} T / \zeta\right)\left[\left(1-\frac{9 R_{i j}}{32 a}\right) \delta_{\alpha \beta}+\left(\frac{3}{32 a}\right) \frac{R_{i j}^{\alpha} R_{i j}^{\beta}}{R_{i j}}\right] .
$$

In this paper, dimensionless quantities are used where length $(l)$, energy $\left(k_{B} T\right)$, and translational friction $\zeta$ $=6 \pi \eta_{s} a$ of chain beads ( $\eta_{s}$ represents the solvent viscosity) are set to unity. It follows that time is reduced by $\zeta l^{2} / k T$ and elongational rate by $k T / \zeta l^{2}$. Any physical quantity within the remainder of this paper refers to dimensionless values using this set of dimensionless units unless otherwise indicated.

Elongational rates spanning a range from 0 to 10 and a time step between $\Delta t=2.5 \times 10^{-4}$ and $1 \times 10^{-3}$ are used within this investigation. Different chain lengths from $N$ $=10$ to $N=94$ are considered (i.e., $N=10,17,22,33,46$, 94). A randomly coiled starting configuration was employed for each simulation. An equilibration period of $2.5 \times 10^{6}$ to $2.5 \times 10^{7}$ time steps, depending on the chain length and elongational rate, was performed before trajectories were saved for analysis. The achievement of steady-state conditions was monitored through the end-to-end-vector, $\vec{R}$, the radius of gyration, $R_{g}$, and the components of the inertia tensor, $\mathbf{T}$. Following equilibration, production runs were performed and the resulting trajectories analyzed. Each production run was between $5 \times 10^{6}$ and $5 \times 10^{7}$ time steps in length depending on the chain length, the elongational rate and the magnitude of the time step.

The remaining sections of this paper investigate the dependence of the steady state size, shape, and the intrinsic elongational viscosity of the chain on elongational rate. Error bars for all plotted data points are smaller than the size of the symbol used unless otherwise indicated. Furthermore, lines connecting data points within figures are an aid to the eye unless otherwise indicated.

\section{RESULTS AND DISCUSSION}

\section{A. Dependencies of chain characteristics on elongational rate}

\section{Size and shape of chains: Projections of bead positions}

Clouds of points corresponding to bead positions relative to the $N=22$ chain's center-of-mass projected onto the $X Y$ plane are plotted in Figs. 1(a)-1(d). Projections are taken at various times throughout the trajectory which employed HI. Figure 1(a) reveals a spherically symmetric coiled distribution of chain beads under a small elongational flow. This distribution deforms and become more and more prolate in the direction of flow when the elongational rate increases. Figure 1(b) shows the cloud of beads at an elongational rate of $\dot{\varepsilon}=0.15$ at the beginning of coil-stretch transition. Significantly more constrained configurations are depicted for elongational flow rates of $\dot{\varepsilon}=0.3$ which lies approximately half way through the coil-stretch transition and $\dot{\varepsilon}=2.0$ (i.e., near the top of the transition region) in Figs. 1(c) and 1(d), respectively. Figures 1(b) and 1(c) reveal that at the transition, the conformation is dumbbell-like with the previously observed single large cloud of points being not only deformed but also separated into several smaller clouds. The

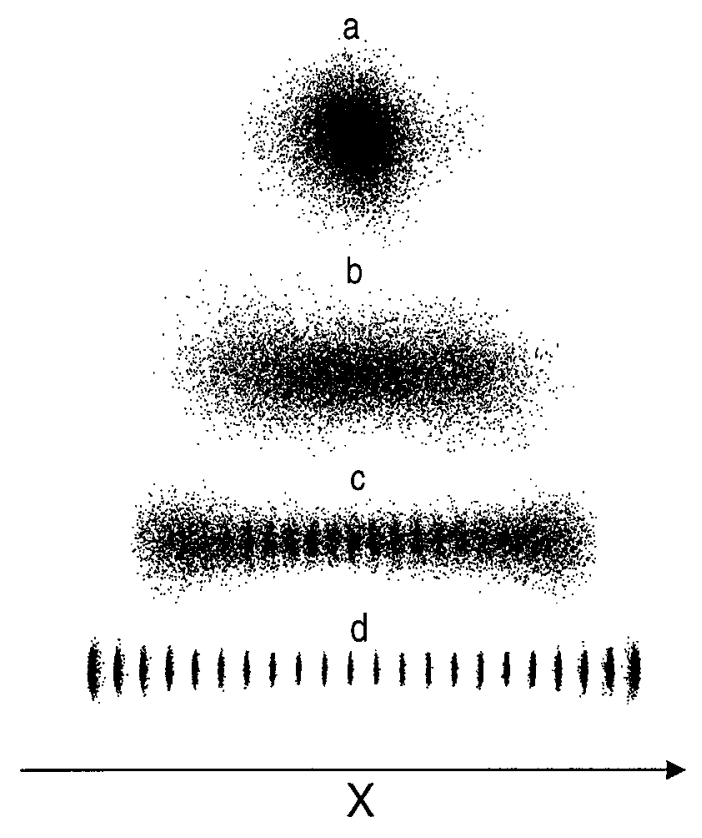

FIG. 1. Clouds of points corresponding to the projections of bead coordinates onto the $X Z$ plane for the $N=22$ chain with $\mathrm{HI}$ at various times throughout the simulation trajectory at different elongational rates: (a) $\dot{\varepsilon}$ $=0.0003$, (b) $\dot{\varepsilon}=0.15$, (c) $\dot{\varepsilon}=0.3$, (d) $\dot{\varepsilon}=2.0$.

smaller clouds become increasingly narrow as the elongational rate is increased with each cloud representing the fluctuations of a single bead. It is clearly seen in Figs. 1(c) and 1(d) that bead fluctuations parallel to the flow direction are less than fluctuations perpendicular to the flow.

\section{Radius of gyration and monomer density distribution}

Figure 2(a) reveals the distribution function of the radius of gyration for the $N=22$ bead chain at different elongational rates. The distribution of the radius of gyration at small elongational rates is similar to that at zero elongational rate. At elongational rates which correspond to the transition region [curve 2, Fig. 2(a)], the distribution becomes significantly broader and is shifted to higher $R_{g}$ values. This broadening indicates an increase in the fluctuations of the chain's size in the vicinity of the transition. At higher rates, the distribution continues to shift to higher $R_{g}$ values but begins to narrow. At very high rates where the chain is close to being completely elongated, the distribution is very sharply peaked. Figure 2(b) illustrates the density distribution function, $\rho(h)$, of monomers as a function of the distance, $h$, from the $N=22$ chain's center-of-mass for a variety of elongational rates. The distribution at small elongational rates is qualitatively similar to that in the absence of flow. At elongational rates which correspond to the coil-stretch transition region, this distribution becomes broader with peaks beginning to appear at various positions along the curve. At the highest rates, the distribution is comprised of a series of separate sharper peaked curves which correspond to the average bead positions of a chain near complete elongation. The increasing ease of identifying peaks within Fig. 2(b) separated by areas of negligible if not zero density as the 

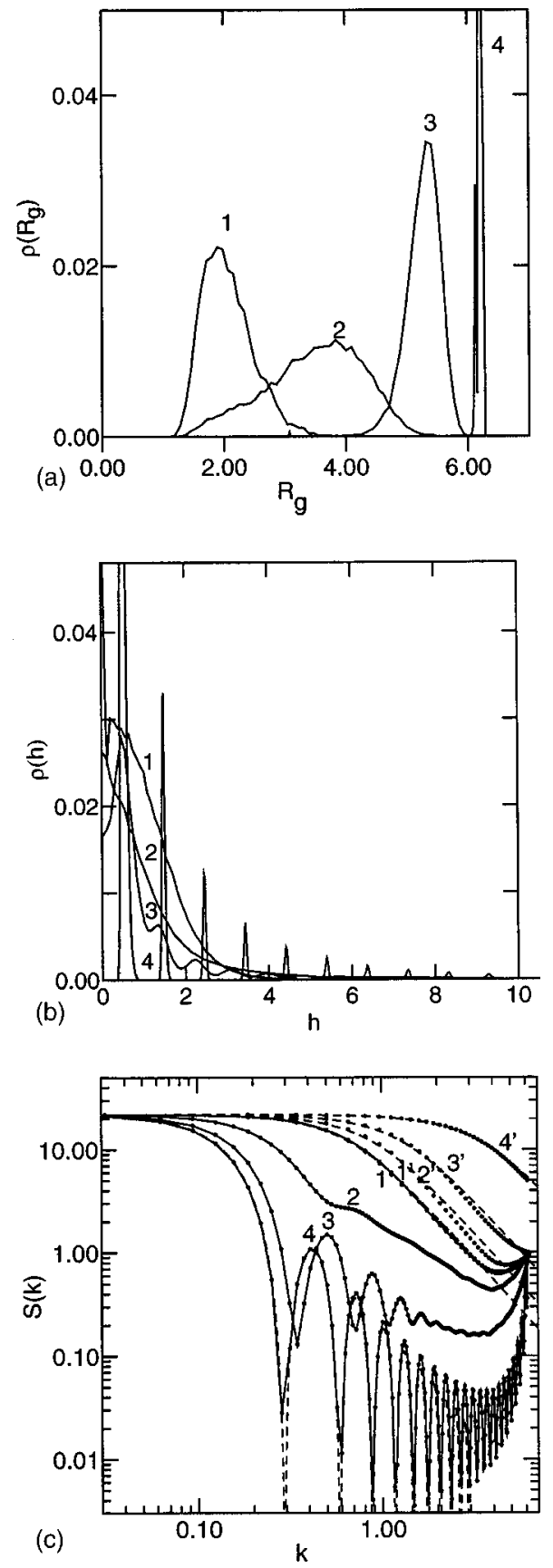

FIG. 2. (a) The distribution function of the radius of gyration of the $N$ $=22$ chain at the elongational rates: (1) $\dot{\varepsilon}=0.0003$, (2) $\dot{\varepsilon}=0.15$, (3) $\dot{\varepsilon}$ $=0.3$, (4) $\dot{\varepsilon}=2.0$. (b) The distribution function of bead positions relative to the center-of-mass for a chain consisting of $N=22$ beads at the same elongational rates as in (a). (c) Structure factor for directions parallel $\left[S_{\text {par }}(k)\right.$; curves $1-4]$ and perpendicular to the flow $\left[S_{\text {per }}(k)\right.$; curves $\left.1^{\prime}-4^{\prime}\right]$ as function of $k$ for the $N=22$ bead chain with HI at the elongational rates used within (a) and (b). The dashed lines running through the $S_{\text {per }}(k)$ data labeled $1^{\prime}-4^{\prime}$ are parameter free fits to the Debye function, Eq. (8b). The dashed line running through the $S_{\text {par }}(k)$ data set labeled 4 reflects a theoretical prediction of a rigid rod (Ref. 50) [see Eq. (8c)].

elongational rate is increased is consistent with the qualitative behavior observed in Figs. 1(a)-1(d) where increasing elongational flow leads to the increased segregation in the clouds.

\section{Structure factor}

The structure factor, $S(\mathbf{k})$, for each stored molecular conformation is calculated along three Cartesian axes, and defined as

$$
S\left(k_{\alpha}\right)=\left\langle\frac{1}{N^{2}}\left|\sum_{j=1}^{N} e^{i k_{\alpha} r_{\alpha}^{j}}\right|^{2}\right\rangle, \quad \alpha=x, y, z .
$$

The angular brackets denote averaging over the entire trajectory at a given elongational rate. In the absence of flow, the calculation is performed for each of the three projections with the quantity $S(k)=1 / 3\left[S_{x}(k)+S_{y}(k)+S_{z}(k)\right]$ being investigated. Under elongational flow, the structure factor along the flow direction, $S_{\mathrm{par}}(k)=S_{x}(k)$, and perpendicular to the flow direction, $S_{\mathrm{per}}(k)=1 / 2\left(S_{y}(k)+S_{z}(k)\right)$, are calculated. The magnitude of the scattering vector, $k$, spans the range $0<k<2 \pi / l$ where $l$ is a bond length. Plots of $S_{\text {par }}(k)$ and $S_{\text {per }}(k)$ at different flow rates are depicted in Fig. 2(c) for the $N=22$ bead chain as function of $k$. The former are labeled as curves 1-4 while the latter are labeled as curves $1^{\prime}-4{ }^{\prime}$. These flow rates are the same as those for the data within Figs. 1, 2(a), and 2(b). The shape of $S_{\text {per }}(k)$ is observed not to change nearly as much as that for $S_{\text {par }}(k)$ when the elongational rate is increased. The shape of $S_{\text {par }}(k)$ and $S_{\text {per }}(k)$ are qualitatively similar to the shape of structure factors $S_{\text {par }}(k)$ and $S_{\text {per }}(k)$ obtained by Pierlioni et al. ${ }^{49}$ for long chains stretched by pair forces applied to their ends. However, all curves are not observed to overlap at high $k$ as reported by Pierlioni et al. ${ }^{49}$ This difference is possibly connected with the different mechanisms by which the external forces act on chain. In the case of Pierlioni et al., pair forces act directly on the chain ends only which may have limited effect on the local structure of the chain's interior. Within this study, the effect of the elongational flow operates directly on each bead. Furthermore, in addition to the stretching forces in operation along the flow direction, there are also compressive forces operating perpendicular to the flow direction. Moreover, for elongational flow the overall extensional forces are stronger in the middle of the chain in comparison to the chain ends.

The shape of $S_{\text {per }}(k)$ is similar at all values of elongational flow $\dot{\varepsilon}$ with the observed slope changes with flow arising from changes in the chain's radius of gyration. $S_{\text {per }}(k)$ is described well by the Debye function (i.e., dashed lines through points labeled $1^{\prime}-4^{\prime}$ )

$$
S_{\mathrm{per}}(k)=\frac{2 N}{A_{\mathrm{per}}^{2}}\left(\exp \left(-A_{\mathrm{per}}\right)-1+A_{\mathrm{per}}\right),
$$

where $A_{\mathrm{per}}=k^{2} R_{g, \mathrm{per}}^{2} / 2$. As the value of $R_{g, \mathrm{per}}^{2}$ is obtained from the simulation as $\left(R_{g y}^{2}+R_{g z}^{2}\right) / 2$, the fit of $S_{\mathrm{per}}(k)$ to Eq. (8b) is parameter free.

The shape of $S_{\text {par }}(k)$ changes significantly with elongation rate. At moderate and high $\dot{\varepsilon}$ values [i.e., lines 3 and 4 in Fig. 2(c)], $S_{\text {par }}(k)$ begins to oscillate dramatically. At the highest elongational flow rate in Fig. 2(c) (i.e., line 4), the oscillating shape for $S_{\text {par }}(k)$ agrees well up to $k<3$ with the theoretical prediction for a structure factor in the limiting case of a rigid rod of length $L$ with $N$ beads uniformly distributed along it ${ }^{50}$ 

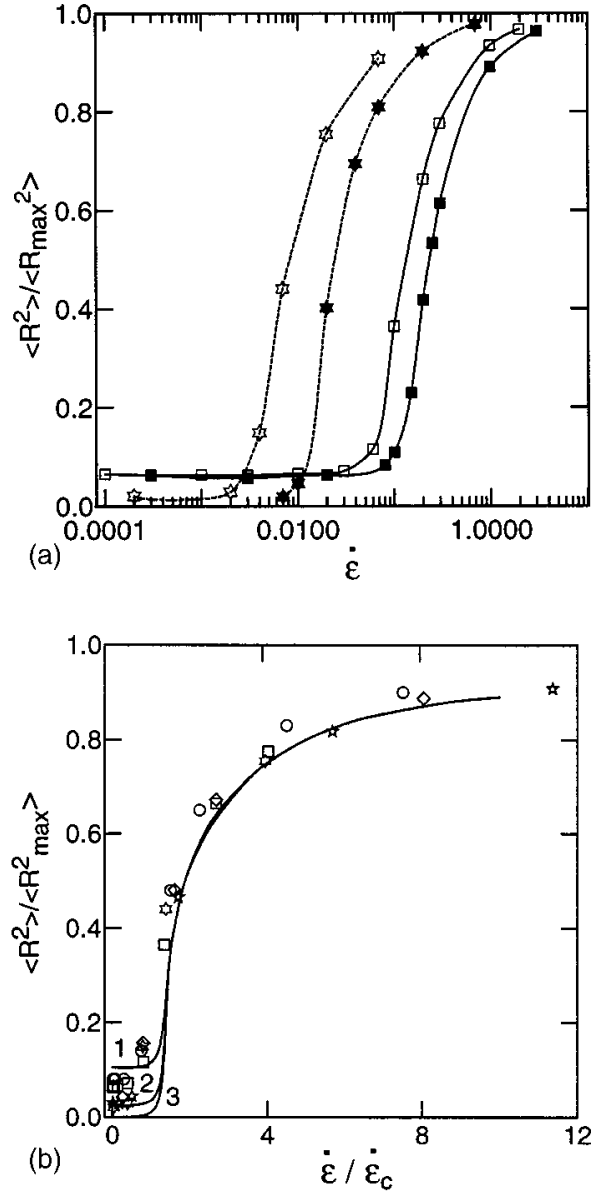

FIG. 3. (a) The elongational rate dependence of the ratio of mean squared end-to-end distance $\left\langle R^{2}\right\rangle$ to its maximum value $\left\langle R_{\max }^{2}\right\rangle$ for a chain of $N$ $=22$ beads with (filled squares) and without (open squares) $\mathrm{HI}$ in addition to $N=94$ beads with (filled six pointed star) and without (open six pointed star) HI. (b) The dependence of $\left\langle R^{2}\right\rangle /\left\langle R_{\max }^{2}\right\rangle$ for chain of $N=10$ (circles), 22 beads (squares), 33 beads (diamonds), 46 beads (five pointed stars), and 94 beads (six pointed stars) on elongational rate (normalized to its critical value) in the absence of HI. The lines are theoretical predictions of Gotlib et al. (Ref. 51) for chains consisting of $N=10,50,250$ beads labeled 1, 2, and 3 , respectively.

$$
S(k)=\frac{2 N}{(k L)^{2}}(1-\cos (k L)) .
$$

Agreement between the data and the theoretical rigid rod prediction becomes worse at higher $k$ where fluctuations begin to dominate the systematic effects as pointed out by Pierleoni et al. (see Fig. 3 of Ref. 49).

\section{Elongational rate dependence of chain size}

The ratio of mean squared end-to-end distance $\left\langle R^{2}\right\rangle$ to its fully stretched value is plotted in Fig. 3(a) as a function of the elongational rate for chains lengths of $N=22$ and $N$ $=94$ with and without HI. At small elongational rates, $\dot{\varepsilon}$ $\ll 1$, chains both with and without HI form coils with a characteristic unperturbed size. Increasing the elongational rate leads to a pronounced stretching of the chain. For chains without HI (open symbols), the steady-state coil-stretch transition is clearly seen in Fig. 3(a) as a very sharp change of $\left\langle R^{2}\right\rangle$ starting near $\dot{\varepsilon}=0.06$ for $N=22$ and near $\dot{\varepsilon}=0.002$ for $N=94$. Hydrodynamic interactions cause this transition

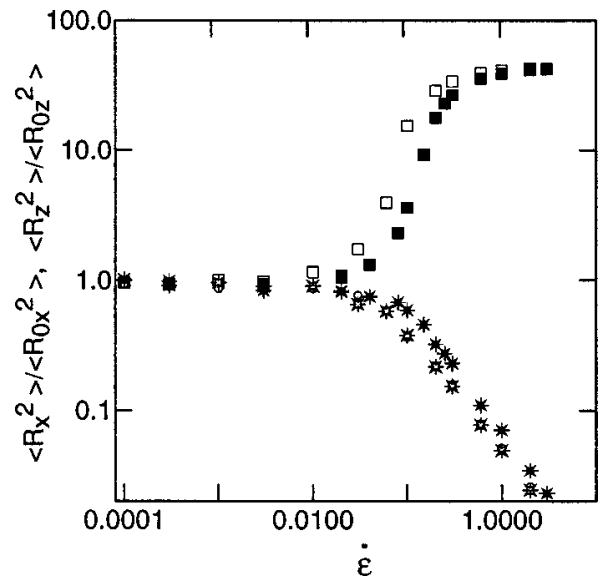

FIG. 4. The elongational rate dependence of the projection of the mean squared end-to-end distance parallel to the flow direction (i.e., $\left\langle R_{x}^{2}\right\rangle$; squares) and perpendicular to the flow (i.e., $\left\langle R_{z}^{2}\right\rangle$; many pointed stars) for the chain with $N=22$ beads without HI (open symbols) and with HI (filled symbols). Both quantities are normalized by their values at zero flow rates.

to occur at slightly higher values of $\dot{\varepsilon}$ starting near 0.1 for the shorter chain with a relatively larger HI-induced shift to a value of $\dot{\varepsilon}=0.01$ observed for the longer chain. This was observed in earlier computational efforts of Cifre and de la Torre $^{34,35}$ for bead-FENE spring chains. Agarwal et al. ${ }^{37}$ obtained this dependence for a bead-rod model at one chain length of $N=100$ in the absence of excluded volume interactions. The extension of the chain as a whole can be characterized also by the means of squared gyration radius $\left\langle R_{g}^{2}\right\rangle$. It is observed that $\left\langle R_{g}^{2}\right\rangle$ behaves similarly and plots of these quantities are therefore not shown.

In Fig. 3(b) the ratio of $\left\langle R^{2}\right\rangle$ to its fully stretched value is plotted as a function of the ratio of the elongational rate to its critical value in the absence of HI. Results for chains of $N=22,33,46$, and 94 beads are plotted and it is clearly seen that all points lie nearly on the same curve. The lines represent the theoretical predictions of this ratio as a function of relative elongational rate for multisegmental bead-spring models with fixed contour length due to Gotlib et al. ${ }^{51}$ in the absence of $\mathrm{HI}$ for chain lengths of $N=10,50$, and 250 beads (curves 1,2 , and 3 , respectively). At $\dot{\varepsilon} / \dot{\varepsilon}_{c}>1$, curves for the different $N$ values vary very little and the good agreement between theory and simulation is observed. This indicates that a multisegmental model with a fixed contour length describes the extension of bead-rod chain within an elongational flow well. A similar theoretical approach was used in the work of Hoffman et al. ${ }^{52}$ These authors found a similar elongational flow dependence on the end-to-end distance for a chain with a fixed contour length and for a freely joint bead-rod model with rigid bond lengths where the constraints were determined via Lagrange multipliers.

\section{Elongational rate dependence of chain shape}

The average shape of the polymer chain as whole in uniaxial elongational flow can be further characterized quantitatively by projections of the mean squared end-to-end distance in the direction of flow, $\left\langle R_{x}^{2}\right\rangle$, and perpendicular to the direction of flow. As the $y$ and $z$ projections are practically 

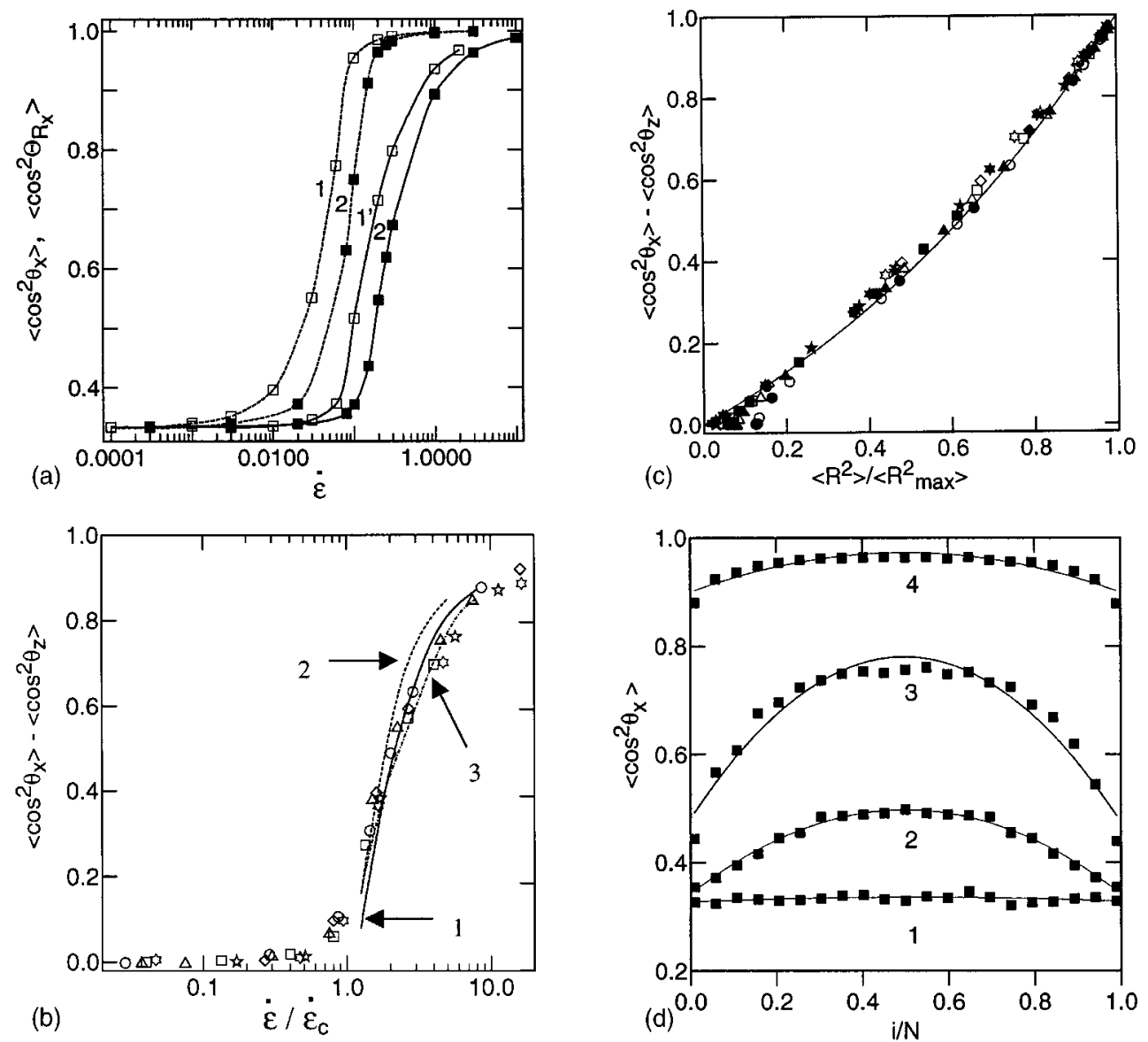

FIG. 5. (a) The elongational rate dependence of the orientation of the $N=22$ chain as a whole (i.e., $\left\langle\cos ^{2} \Theta_{R x}\right\rangle$; dashed lines labeled 1 and 2$)$ and the orientation of chain bonds (i.e., $\left\langle\cos ^{2} \theta_{x}\right\rangle$; solid lines labeled 1' and $\left.2^{\prime}\right)$ in the direction of flow. Open symbols represent a chain without HI while filled symbols represent a chain with HI. (b) The elongational rate dependence of the normalized birefringence (i.e., $\left.\left\langle\cos ^{2} \theta_{x}\right\rangle-\left\langle\cos ^{2} \theta_{z}\right\rangle\right)(\operatorname{circles}, N=10$; triangles, $N=17$; squares, $N=22$; diamonds, $N=33$; five pointed stars, $N=46$; and six pointed stars, $N=94$ ). The line labeled 1 represents the theory of Gotlib et al. (Ref. 51). The lines labeled 2 and 3 represent experimental results cited in Ref. 51 for polystyrene of molecular weight $2.07 \times 10^{6}$ daltons and 4.2 $\times 10^{6}$ daltons correspondingly. (c) The dependence of the relative mean squared end-to-end distance of chain on normalized birefringence (i.e., $\left\langle\cos ^{2} \theta_{x}\right\rangle$ $-\left\langle\cos ^{2} \theta_{z}\right\rangle$ ) for different chain lengths (circles, $N=10$; triangles, $N=17$; squares, $N=22$; diamonds, $N=33$, five pointed star, $N=46$; six pointed star, $N$ =94). Open symbols represent simulations without $\mathrm{HI}$ and filled symbols for those performed with $\mathrm{HI}$. The solid line represents the theoretical prediction of Kuhn-Grun (Ref. 53). (d) The mean squared cosine of the angle between each bond and the flow direction as a function of the $N$-normalized bond number along the chain backbone at the elongational rates (1) $\dot{\varepsilon}=0.0003$, (2) $\dot{\varepsilon}=0.15$, (3) $\dot{\varepsilon}=0.3$, and (4) $\dot{\varepsilon}=2.0$. The solid lines reflect the best fit parabola at each value of $\dot{\varepsilon}$.

indistinguishable, only $\left\langle R_{z}^{2}\right\rangle$ is presented as the projection perpendicular to the direction of flow. Values of $\left\langle R_{x}^{2}\right\rangle$ and $\left\langle R_{z}^{2}\right\rangle$ for a chain length of $N=22$ are shown in Fig. 4 both in the absence of HI (open symbols) and in the presence of HI (filled symbols) normalized by their zero flow values. The overall behavior of $\left\langle R_{x}^{2}\right\rangle$ and $\left\langle R_{z}^{2}\right\rangle$ as a function of elongational rate is qualitatively similar for cases where HI are invoked and where they are neglected. At small elongational rates, all values of $\left\langle R_{x}^{2}\right\rangle$ and $\left\langle R_{z}^{2}\right\rangle$ are similar and independent of $\dot{\varepsilon}$. At higher elongational rates, $\left\langle R_{x}^{2}\right\rangle$ begins to increase and $\left\langle R_{z}^{2}\right\rangle$ begins to decrease towards a value near zero at high elongational rates. The behavior of $\left\langle R_{x}^{2}\right\rangle$ is similar to that of the mean squared end-to-end distance within Fig. 3(a). At high $\dot{\varepsilon}$ where the chain is nearly completely extended, $\left\langle R_{x}^{2}\right\rangle$ plateaus at a value near the contour length of chain. The decrease of $\left\langle R_{z}^{2}\right\rangle$ with $\dot{\varepsilon}$ can be described by a power law for the chain with and without $\mathrm{HI}$ with exponents of -0.97 and -0.96 , respectively.

The shape of the chain as a whole can also be character- ized by the projections of the squared radii of gyration, $\left\langle R_{g x}^{2}\right\rangle$ and $\left\langle R_{g z}^{2}\right\rangle$. It was observed that $\left\langle R_{g x}^{2}\right\rangle$ and $\left\langle R_{g z}^{2}\right\rangle$ behave similar to $\left\langle R_{x}^{2}\right\rangle$ and $\left\langle R_{z}^{2}\right\rangle$ and plots of these quantities are not shown. The decrease of $\left\langle R_{g z}^{2}\right\rangle$ with $\dot{\varepsilon}$ can also be described by a power law for the chain with and without HI with exponents of -0.98 and -0.96 , respectively.

\section{Global and local orientation of the chain, and chain birefringence}

The average orientation of the chain as whole relative to the direction of elongational flow can be characterized by the angle, $\Theta_{R x}$ or by $\left\langle\cos ^{2} \Theta_{R x}\right\rangle=\left\langle R_{x}^{2}\right\rangle /\left\langle R^{2}\right\rangle$ which is plotted in Fig. 5(a) for $N=22$ (dashed lines) for simulations in the absence of $\mathrm{HI}$ and in the presence of $\mathrm{HI}$ (i.e., lines 1 and 2, respectively). Both curves illustrate similar features. At values of $\dot{\varepsilon}<0.003$, the value of $\left\langle\cos ^{2} \Theta_{R x}\right\rangle$ for a chain without $\mathrm{HI}$ is $1 / 3$, denoting random chain orientation, and is virtually independent of elongational rate. As $\dot{\varepsilon}$ increases, the inclina- 
tion of the chains depicted by both curves (i.e., with and without $\mathrm{HI}$ ) undergoes a transition to a plateau of nearly 1 on the $y$ axis which corresponds to complete alignment of the chain as a whole along the direction of flow. Hydrodynamic interactions tend only to delay the onset of this step transition and, again, this behavior is qualitatively similar to that of the mean squared end-to-end distance within Fig. 3(a). However, the increase of $\left\langle R_{x}^{2}\right\rangle /\left\langle R^{2}\right\rangle$ in Fig. 5(a) occurs earlier than that for $\left\langle R^{2}\right\rangle$ for the same chain length (i.e., $N$ $=22$ ) for the cases with and without HI [Fig. 3(a), open and filled squares]. Similar behavior was observed for all other chain lengths. The orientation of the chain as a whole in elongational flow can also be characterized by $\left\langle\cos ^{2} \Theta_{R g x}\right\rangle$ $=\left\langle R_{g x}^{2}\right\rangle /\left\langle R_{g}^{2}\right\rangle$. It is observed that $\left\langle\cos ^{2} \Theta_{R g x}\right\rangle$ behaves similar to $\left\langle\cos ^{2} \Theta_{R x}\right\rangle$ and plots of these quantities are therefore not illustrated.

A similar analysis is depicted in Fig. 5(a) for $N=22$ (solid lines) for a local orientational angle, $\theta_{x}$, representing the average angle between the flow direction and the individual rigid bonds of the chain. This angle is plotted in Fig. $5(\mathrm{a})$ as $\left\langle\cos ^{2} \theta_{x}\right\rangle$ (i.e., lines $1^{\prime}$ and $2^{\prime}$, respectively) which is computed as the ratio $\left\langle l_{x}^{2}\right\rangle /\left\langle l^{2}\right\rangle$ where $\left\langle l_{x}^{2}\right\rangle$ is the mean squared $x$ projection of a chain bond averaged over all chain monomers. $\left\langle l^{2}\right\rangle$ equals a constant for a bead-rod chain which is unity within the current set of dimensionless units. The general shape of the curves representing the average orientation of the chain as a whole and the curves representing the local orientation of the chain are similar. However, a sharp transition from randomly oriented bonds (i.e., $\left.\left\langle\cos ^{2} \theta_{x}\right\rangle=1 / 3\right)$ to completely oriented bonds $\left\langle\cos ^{2} \theta_{x}\right\rangle=1$ ) occurs at a significantly larger $\dot{\varepsilon}$ value than for the orientation of the chain as a whole. Once again HI cause the onset of the transition to be delayed to higher elongational flow. This implies different values of $\dot{\varepsilon}_{c}$ would be predicted depending on whether the onset of the coil-stretch transition was detected by the step change in $\left\langle\cos ^{2} \theta_{x}\right\rangle$, which is connected with birefringence, or by $\left\langle\cos ^{2} \Theta_{R x}\right\rangle$. Additionally, the onset of the local orientation [i.e., Fig. 5(a), lines $1^{\prime}$ and 2'] is nearly the same as the onset of the transition in the mean squared end-to-end data within Fig. 3(a) for the same $N$ $=22$ chain (i.e., open and filled squares for chain without and with HI, respectively).

By defining a $\left\langle\cos ^{2} \theta_{z}\right\rangle$ in a manner analogous to $\left\langle\cos ^{2} \theta_{x}\right\rangle$, the dependence of $\left\langle\cos ^{2} \theta_{x}\right\rangle-\left\langle\cos ^{2} \theta_{z}\right\rangle$, which is the normalized chain birefringence, on the relative elongational rate $\dot{\varepsilon} / \dot{\varepsilon}_{c}$ is illustrated in Fig. 5(b) for the case of $N=22,33$, 46, and 94 without HI. Also included within this figure as the line labeled 1 is the theoretical prediction of Gotlib et al. ${ }^{51}$ for multisegmental chains of fixed contour length without HI under elongational flow. The dashed lines represent experimental polystyrene data cited in Ref. 51 of molecular weights of $2.07 \times 10^{6}$ (i.e., line 2 ) and $4.2 \times 10^{6}$ (i.e., line 3 ) daltons. The agreement between the simulation results with the theoretical predictions and the experimental findings is observed to be good.

Figure 5(c) is a cross plot depicting the dependence of the normalized value of the birefringence versus normalized chain extension defined as $\left\langle R^{2}\right\rangle /\left\langle R_{\max }^{2}\right\rangle$. The birefringence is defined as for Fig. 5(b). This plot offers insight into how flow induced changes in the local orientation of monomers correlates with the chain extension. The data within this figure reveals $\left\langle\cos ^{2} \theta_{x}\right\rangle-\left\langle\cos ^{2} \theta_{z}\right\rangle$ and $R_{\text {norm }}^{2}=\left\langle R^{2}\right\rangle /\left\langle R_{\text {max }}^{2}\right\rangle$ are strongly correlated. This behavior is compared with the theoretical prediction of Kuhn and Grun ${ }^{53}$ relating birefrigence to relative chain extension which is illustrated as a solid line within Fig. 5(c). This curve is a numerical approximation of the inverse Langevin function and has the form $\Delta n / n_{0}$ $=0.6 R_{\text {norm }}^{2}+0.2 R_{\text {norm }}^{4}+0.2 R_{\text {norm. }}^{6}$ Overall agreement is excellent with slight differences observed only for short chains consisting of $N=10$ (circles) and $N=17$ beads (triangles) at small values of chain extension.

Figure 5(d) illustrates the mean squared cosine of the angle between each bond and the flow direction as function of bond number $i$ along the chain backbone (normalized by $N)$ at different elongational rates. At very low shear rates, all bond orientations are near $1 / 3$ reflecting the equal probability of orientation of each bond in the $x, y$, and $z$ directions. When flow increases, the middle bonds begin to be more and more oriented in the direction of flow. At the same time, the orientation of bonds close to the chain ends is only slightly affected by flow even at elongational rates close to the middle of the coil-stretch transition. The general profile of curves 2 and 3 within Fig. 5(d) is similar to the theoretically predicted parabolic profile ${ }^{54}$ with some deviation noticed near the chain interior. The observation that the maximum extent of local orientation and elongation is found near the middle of the chain is consistent with experimental efforts of chain scission within elongational flow ${ }^{2}$ where breaks always occur near the chain center. Increasing elongational flow further increases all bond orientation with an accompanying reduction in the difference between midchain and chain end orientation.

\section{Intrinsic elongational viscosity}

The primary rheological property of interest in this study is the stress tensor, given by the Kramers expression ${ }^{48}$

$$
\tau=-\eta_{0} \dot{\varepsilon}-n\langle\mathbf{R F}\rangle+n \mathbf{I},
$$

where $\dot{\varepsilon}$ is the rate-of-strain tensor and $\eta_{0}$ is the elongational viscosity of the solvent. For simple elongational flow, $\dot{\varepsilon}_{x x}=$ $-2 \dot{\varepsilon}_{y y}=-2 \dot{\varepsilon}_{z z}=\dot{\varepsilon}$ and all off diagonal components of the rate-of-strain tensor are zero. Here the $n$ is the number density of chains, $\mathbf{R F}$ is the virial tensor for a single chain. The elongational viscosity is expressed in terms of the elongational stress as

$$
\eta_{\mathrm{el}}=-\frac{\tau_{x x}-\tau_{z z}}{\dot{\varepsilon}}=\eta_{0}+n \frac{\mathbf{R}_{x} \mathbf{F}_{x}-\mathbf{R}_{z} \mathbf{F}_{z}}{\dot{\varepsilon}},
$$

and the corresponding intrinsic elongational viscosity is calculated using Eq. (10a) as

$$
\left[\eta_{\mathrm{el}}\right]=\left[\frac{\left(\eta_{\mathrm{el}}-\eta_{0}\right)}{n \eta_{0}}\right] \text {. }
$$

The intrinsic elongational viscosity $\left[\eta_{\mathrm{el}}\right]$ afforded by simulations with and without $\mathrm{HI}$ is plotted in Fig. 6(a) for the $N$ $=22$ chain as a function of elongational rate. Each set of data 

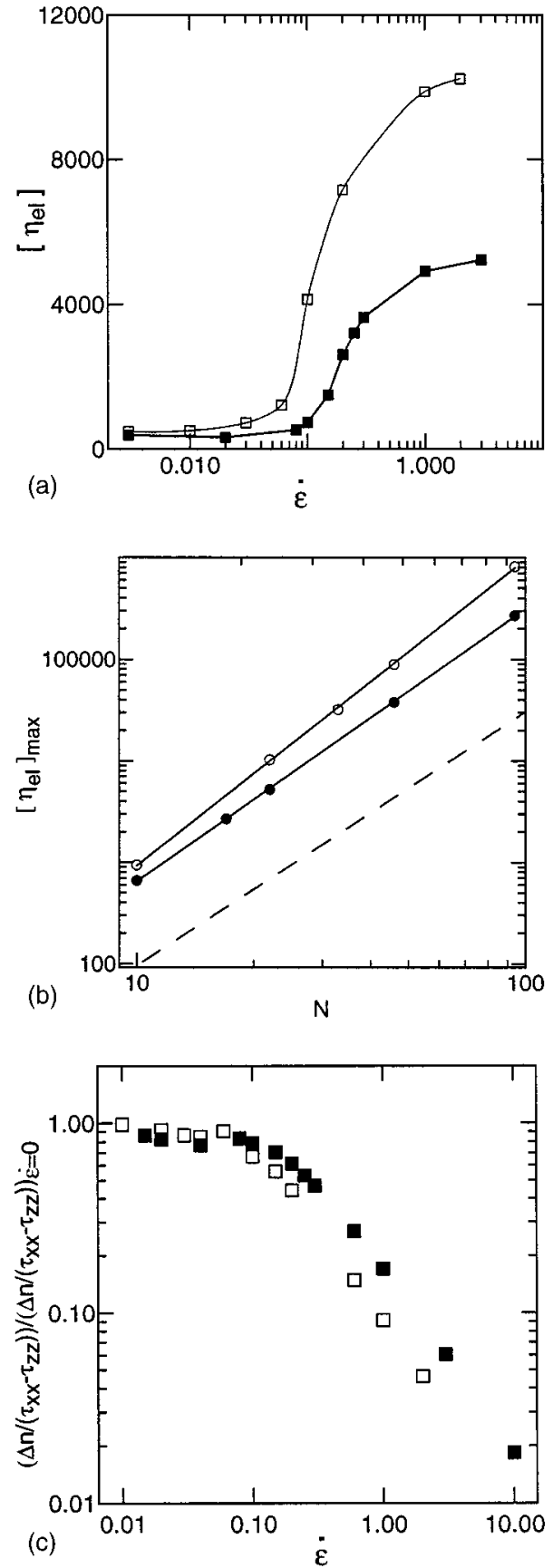

FIG. 6. (a) The elongational rate dependence of the intrinsic elongational viscosity for a $N=22$ chain. Open symbols reflect simulations performed without HI with closed symbols reflecting simulations invoking HI. (b) The dependence of the plateau values of the intrinsic elongational viscosity, $\left[\eta_{\mathrm{el}}\right]_{\max }$, on chain length, $N$. Open symbols reflect simulations performed without HI with closed symbols reflecting simulations invoking HI. The solid lines are best fits implying a power law relationship between $\left[\eta_{\mathrm{el}}\right]_{\max }$ and $N$. The dashed line represents the theoretical dependence of $\left[\eta_{\mathrm{el}}\right]_{\max }$ on $N$ for a rigid rod with HI from Ref. 48. (c) The ratio of the birefringence to the difference in the stress components parallel and perpendicular to the flow directions [i.e., stress-optical relationship (Ref. 48)] as a function of the flow rate. The data is for a chain of $N=22$ beads. Open symbols reflect simulations performed without $\mathrm{HI}$ with closed symbols reflecting simulations invoking HI. The data on the $y$ axis has been normalized by its value at an elongational rate of zero.

reveals a transition from an unperturbed coil at low elongational rates to a highly stretched rodlike conformation at high elongational rates. Hydrodynamic interactions are observed to displace occurrence of this transition to higher elonga- tional rates as in earlier figures for chain extension and chain orientation. At high elongational rates, the plateau value of $\left[\eta_{\mathrm{el}}\right]$ for the model with $\mathrm{HI}$ is observed [Fig. 6(a)] to be significantly lower than $\left[\eta_{\mathrm{el}}\right]$ values without HI. This observation is expected since HI between chain beads act to decrease chain friction and hence viscosity relative to the case where HI are absent. ${ }^{48,55}$

The simulated values of $\left[\eta_{\mathrm{el}}\right]$ without HI were compared with the theoretical predictions of Hassager ${ }^{56}$ in the following two limiting cases:

$$
\begin{aligned}
& {\left[\eta_{\mathrm{el}}\right]=\left[\eta_{\mathrm{el}}\right]^{\min }\left(1+\frac{N^{2} \dot{\varepsilon}}{90}+\cdots\right) \quad \text { at small } \dot{\varepsilon},} \\
& {\left[\eta_{\mathrm{el}}\right]=\left[\eta_{\mathrm{el}}\right]^{\max }\left(1-\frac{24}{N^{2} \dot{\varepsilon}}-\cdots\right) \text { at large } \dot{\varepsilon},}
\end{aligned}
$$

where $\left[\eta_{\mathrm{el}}\right]^{\min }$ and $\left[\eta_{\mathrm{e}}\right]^{\max }$ are limiting values of characteristic elongational intrinsic viscosity at low and high flow rates, respectively. After truncating Eqs. (11a) and (11b) to the first two terms as shown above within the parentheses on the right-hand side of each equation, it is noted (figure not shown) that Eq. (11a) describes the behavior of $\left[\eta_{\mathrm{el}}\right]$ well at small $\dot{\varepsilon}$ before the transition. Equation (11b) describes the data for chains with and without HI well from the high $\dot{\varepsilon}$ plateau to the middle of the transition (figure not shown). A similar conclusion was also made both by Doyle et al. ${ }^{25}$ as well as Agarwal et al. ${ }^{37}$ for a bead-rod model without HI.

The plateau values of the intrinsic elongational viscosity, $\left[\eta_{\mathrm{el}}\right]_{\max }$, at large elongational rates for chains of length $N$ $=10$ to $N=94$ are plotted on Fig. 6(b) as function of chain length $N$ on a double log scale. Filled symbols correspond to simulations where $\mathrm{HI}$ are invoked with open symbols representing simulations performed in the absence of HI. The lines running through the symbols indicate $\left[\eta_{\mathrm{el}}\right]_{\max } \sim N^{3}$ for simulations performed in the absence of $\mathrm{HI}$ and $\left[\eta_{\mathrm{el}}\right]_{\max }$ $\sim N^{2.67}$ for simulations performed in the presence of HI. These dependencies can be compared with theoretical results for chains without and with HI. For a freely draining chain, $\left[\eta_{\mathrm{el}}\right]_{\max }$ has been shown to scale as $N^{3}$ in the theoretical efforts of Larson et al. ${ }^{26}$ The slope obtained from the simulation which invoked $\mathrm{HI}$ is in reasonable agreement with the average slope of the curve for a rigid rod with HI [i.e., dashed line within Fig. 6(b)] generated from data tabulated in the monograph of Bird et al. ${ }^{48}$

\section{Stress-optical law}

The stress-optical relationship predicts that the index of refraction tensor should be proportional to the stress tensor. ${ }^{48}$ For the case of uniaxial elongational flow, this rule implies the chain birefringence is proportional to the difference in the stress components parallel and perpendicular to the flow directions. This can be written as

$$
\frac{\Delta n}{\tau_{x x}-\tau_{z z}}=\text { const. }
$$

Figure 6(c) reveals this law is valid both for chain models without and with HI before the onset of the coil-stretch transition. Values on the $y$ axis of this plot have been normalized by their values at zero elongation rate. Within the 


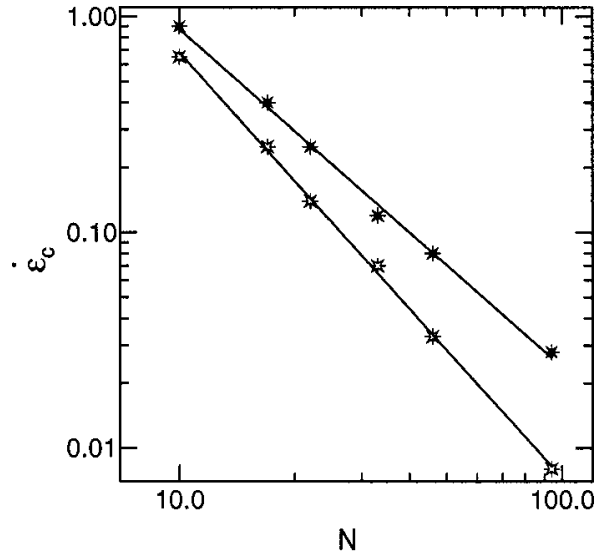

FIG. 7. The dependence of the critical value of the elongational rate on chain length for chains without HI (open symbols) and with HI (filled symbols) drawn from the mean squared end-to-end data for chains of $N=10,17$, $22,33,46$, and 94 . Solid lines reflect best fits implying a power law relationship between the critical value of the elongational rate and the chain length.

transition region, the difference in the stress components increases faster than the birefringence leading to an overall decrease in the ratio within Eq. (12). This decrease is nearly linear on this plot's $\log -\log$ scale Exponents for the power law describing this decay are -0.96 and -0.97 for a chain with and without $\mathrm{HI}$ which corresponds well with the power law decays of the perpendicular projections of the end-to-end distance and the radius of gyration. Similar results were obtained by Wiest ${ }^{57}$ for models of polymer chains composed of $N=5,10$, and 20 FENE springs under the $\mathrm{P}-\mathrm{dG}$ approximation and by Doyle et al. ${ }^{25}$ for a Kramers bead-rod chains without HI. Wiest interpreted the result in terms of the conformational properties of the chain. He revealed that for a FENE chain the moment of inertia of the chain about the flow axis undergoes a transition at smaller values of elongational flow than the end-to-end distance. ${ }^{14,57}$ Wiest attributed this observed flow induced decrease of the chain size perpendicular to the flow direction to the orientation of the individual bond vectors. However, Fig. 5(a) within this paper reveals the onset of increased orientation of the bonds within the bead-rod model employed within this study occurs at higher values of elongational flow relative to analogous quantities for whole-chain orientation. This implies that the decrease of the perpendicular component of the radius of gyration and the perpendicular component of the end-to-end vector in bead-rod model is initially due to increased orientation of the chain as a whole rather than increased orientation of the individual bonds.

\section{B. Dependencies of transition rate on chain length}

The dependence of the mean square end-to-end distance on $\dot{\varepsilon}$ has been calculated for chains of length $N=10,17,22$, 33, 46 and $N=94$ with and without HI. These data allow quantitative estimations of the critical value of elongational rate, $\dot{\varepsilon}_{c}$, to be made and its dependence on chain length to be investigated. In Fig. 7, the critical value, $\dot{\varepsilon}_{c}$, is plotted as function of chain length $N$ for simulations performed with and without HI. These $\dot{\varepsilon}_{c}$ are obtained from mean squared end-to-end versus $\dot{\varepsilon}$ data. The same procedure was done to obtain $\dot{\varepsilon}_{c}$ at each $N$ (plot not shown) from the dependence of the average local chain orientation $\left\langle\cos ^{2} \theta_{x}\right\rangle$, of chain monomers on $\dot{\varepsilon}$. The resulting power law parameters (i.e., $\dot{\varepsilon}_{c}$ $\sim \kappa N^{-\gamma}$ ) extracted from best-fit lines of the double logarithmic plots are given in Table I. The ratio of prefactors, $\kappa$, for bead-rod chains without and with HI obtained from chain extension and from the local orientation is close to 2. This is similar to the analogous value of this ratio for a FENE chain. ${ }^{35}$ The value of the prefactor for a bead-rod model with and without $\mathrm{HI}$ is approximately twice that for a FENE chain ${ }^{35}$ with and without HI, respectively. However, this difference could be linked to the portion (i.e., onset or midpoint) of the transition used to define $\dot{\varepsilon}_{c}$. The slopes obtained from chain extension and from the local orientation of the monomers, respectively, are very similar (i.e., 1.97 and 1.96 without $\mathrm{HI}$ and 1.56 and 1.55 with HI). They agree well with slopes for bead-FENE spring simulations of de la Torre without $\mathrm{HI}$ and with EV and with $\mathrm{HI}$ and $\mathrm{EV}$ (2.00 and 1.55, respectively) and to theoretical predictions ${ }^{2}$ (2.0 and 1.5 for chain with and without HI, respectively) obtained on the basis of the Rouse and Zimm models, respectively. Furthermore, the exponent of around 1.50 to 1.56 for simulations using $\mathrm{HI}$ is in reasonable agreement with available experimental data. ${ }^{2}$

\section{SUMMARY}

Brownian dynamics simulations of the statistical and rheological properties of a bead-rod model of a polymer chain under elongational flow of different lengths with excluded volume have been performed. Both chains with and

TABLE I. Parameters $\kappa$ and $\gamma$ extracted from fitting the data in Fig. 7 to $\dot{\varepsilon}_{c} \approx \kappa N^{-\gamma}$.

\begin{tabular}{lcc}
\hline \multicolumn{1}{c}{ Model } & $\left\langle R^{2}\right\rangle /\left\langle R_{\max }^{2}\right\rangle$ on $N$ & $\left\langle\cos ^{2} \theta_{x}\right\rangle$ on $N$ \\
\hline Bead-rod chain with HI, & $\kappa=31.8 \pm 1.3$ & $\kappa=35.9 \pm 1.2$ \\
(Fig. 7 filled symbols) & $\gamma=1.56 \pm 0.04$ & $\gamma=1.55 \pm 0.03$ \\
Bead-rod chain without HI, & $\kappa=63.1 \pm 1.1$ & $\kappa=73.5 \pm 1.2$ \\
(Fig. 7 open symbols) & $\gamma=1.97 \pm 0.03$ & $\gamma=1.96 \pm 0.03$ \\
Bead-spring FENE chain & $\kappa=14.1 \pm 1.1$ & \\
with HI (Ref. 35) & $\gamma=1.55 \pm 0.03$ & \\
Bead-spring FENE chain & $\kappa=28.6 \pm 0.3$ & \\
without HI (Ref. 35) & $\gamma=2.005 \pm 0.011$ & \\
\hline \hline
\end{tabular}


without HI were studied and compared at different elongational rates. The distribution function of the radius of gyration and the distribution of monomers around the center of inertia of the chain were obtained at different flow rates. These distribution functions significantly broaden near $\dot{\varepsilon}_{c}$ which corresponds to increased fluctuations of the chain size in this region. Structure factors for directions parallel and perpendicular to the direction of flow were calculated. The perpendicular component is described well by the Debye function without any adjustable parameters. The parallel component oscillates significantly at large extensions in accordance with previous results of Pierleoni et al. ${ }^{49}$ for a chain extended by pairwise forces and with the theoretical formula of Onuki ${ }^{50}$ in the limit of a rigid rod. It was shown that bond orientation depends significantly on their position along the chain at intermediate flow rates. The profile of bond orientation as a function of bond number along the chain is nearly parabolic with the maximum extent of orientation occurring in the middle of the chain. This result is in agreement with the theoretical prediction of Henyey and Rabin $^{54}$ and the experimental observation that linear chains under elongational flow are observed to break near the middle of the chain. A "flat-topped" deviation from the parabolic shape is observed near the chain center. Both the average chain extension and chain orientation have been calculated as a function of $\dot{\varepsilon}$. It is observed that critical values of the elongational rates obtained from chain extension as a whole and from the local orientation of the monomers during the coil-stretch transition are nearly the same [see Fig. 5(c)]. At the same time, the orientation of the chain as whole occurs at smaller values of $\dot{\varepsilon}$ especially for longer chains. Inclusion of HI leads to a shift of the coil-stretch transition for all these conformational and orientational characteristics to higher values of $\dot{\varepsilon}$. It was found that the limiting intrinsic elongational viscosity at high elongational rate is smaller for the chain with HI than for the chain without HI. The dependence of critical elongation rate $\dot{\varepsilon}_{c}$ on chain length, $N$ was calculated for the first time for a bead-rod model with fluctuating HI. Despite the different critical value of $\dot{\varepsilon}_{c}$ for the bead-rod chain and the FENE chain, both models reveal nearly the same scaling behavior as a function of chain length which is in good agreement with the theoretical predictions of the Rouse and Zimm models.

\section{ACKNOWLEDGMENTS}

The authors acknowledge the EPSRC for financial support of this study (GR/M64215). This work is partly supported by INTAS 00-00712 and the ESF program SUPERNET.

\footnotetext{
${ }^{1}$ F. C. Frank, A. Keller, and M. R. Mackley, Polymer 12, 467 (1971).

${ }^{2}$ Flexible Polymer Chain Dynamics in Elongational Flow. Theory and Experiment, edited by T. D. Nguyen and H. H. Kausch (Springer-Verlag, Heidelberg, 1999).

${ }^{3}$ M. G. Miles and A. Keller, Polymer 21, 1295 (1980); K. Gardener, E. P. Pike, M. J. Miles, A. Keller, and K. Tanaka, ibid. 23, 1435 (1982); A. Keller and J. A. Odell, Colloid Polym. Sci. 263, 181 (1985); J. A. Odell, A. Keller, and Y. Rabin, J. Chem. Phys. 88, 4022 (1988).

${ }^{4}$ P. N. Dunlap and L. G. Leal, J. Non-Newtonian Fluid Mech. 16, 5 (1987).
}

${ }^{5}$ V. Tirtaamadja and V. Sridhar, J. Non-Newtonian Fluid Mech. 37, 1081 (1993).

${ }^{6}$ S. H. Spiegelberg and G. H. McKinley, J. Non-Newtonian Fluid Mech. 67, 49 (1996).

${ }^{7}$ T. T. Perkins, D. E. Smith, and S. Chu, Science 276, 2016 (1997); D. E. Smith and S. Chu, ibid. 281, 1335 (1998).

${ }^{8}$ P. G. De Gennes, J. Chem. Phys. 60, 5030 (1974).

${ }^{9}$ A. Peterlin, J. Polym. Sci., Part B: Polym. Lett. 4, 287 (1966).

${ }^{10}$ E. J. Hinch, Proceedings Internationaux Centre de la Recherche Scientifique (Editions du Centre National de la Recherche Scientifique, Paris, 1974), No. 233, page 241.

${ }^{11}$ Yu. V. Brestkin, Acta Polym. 38, 470 (1987).

${ }^{12}$ J. J. Magda, R. G. Larson, and M. E. Mackay, J. Chem. Phys. 89, 2504 (1988).

${ }^{13}$ M. Fixman, J. Chem. Phys. 42, 3831 (1965); C. W. Pyun and M. Fixman, ibid. 42, 3838 (1965); 44, 2107 (1966); M. Fixman, ibid. 45, 793 (1966).

${ }^{14}$ J. W. Wiest, L. E. Wedgewood, and R. B. Bird, J. Chem. Phys. 90, 587 (1989).

${ }^{15}$ J.-P. Ryckaert and A. Bellemans, Chem. Phys. Lett. 30, 123 (1975).

${ }^{16}$ Yu. Ya. Gotlib, N. K. Balabaev, A. A. Darinskii, and I. M. Neelov, Polym. Sci. U.S.S.R. A20, 2194 (1978); Macromolecules 13, 602 (1980).

${ }^{17}$ M. N. Lukjanov, A. A. Darinskii, Yu. Ya. Gotlib, and I. M. Neelov, Polym. Sci. U.S.S.R. A27, 1506 (1985).

${ }^{18}$ D. Acierno, G. Titomantio, and G. Marrucci, J. Polym. Sci., Polym. Phys. Ed. 12, 2177 (1974).

${ }^{19}$ A. Rey, J. J. Freire, and J. García de la Torre, Macromolecules 20, 2385 (1987).

${ }^{20}$ A. A. Darinskii, I. M. Neelov, and L. I. Klushin, "Brownian dynamics of polymer chain models with rigid bonds," in Mathematical Methods for Polymers (SCBI, Pushchino, 1982), p. 87.

${ }^{21}$ I. M. Neelov and J. H. R. Clarke, Macromol. Symp. 81, 55 (1994).

${ }^{22}$ J. M. Rallison and E. J. Hinch, J. Non-Newtonian Fluid Mech. 29, 37 (1988).

${ }^{23}$ E. J. Hinch, J. Non-Newtonian Fluid Mech. 54, 209 (1994).

${ }^{24}$ T. W. Liu, J. Chem. Phys. 90, 5826 (1989).

${ }^{25}$ P. Doyle, E. S. G. Shaqfen, and A. P. Gast, J. Fluid Mech. 334, 251 (1997).

${ }^{26}$ R. G. Larson, H. Hu, D. E. Smith, and S. Chu, J. Rheol. 43, 267 (1999).

${ }^{27}$ A. A. Darinskii and M. G. Saphiannikova, J. Non-Cryst. Solids 172-174, 932 (1994).

${ }^{28}$ A. A. Darinskii, M. G. Saphiannikova, and I. Emri, Polym. Sci. U.S.S.R. 37, 1502 (1995).

${ }^{29}$ M. G. Saphiannikova, A. A. Darinskii, and N. E. Dyakonova, Macromol. Theory Simul. 9, 270 (2000).

${ }^{30}$ J. J. López Cascales and J. García de la Torre, J. Chem. Phys. 95, 9384 (1991); 97, 4549 (1992).

${ }^{31}$ J. J. López Cascales and J. García de la Torre, J. Non-Cryst. Solids 172174, 823 (1994).

${ }^{32}$ J. J. López Cascales, F. G. Díaz, and J. García de la Torre, Polymer 36, 345 (1995).

${ }^{33}$ K. D. Knudsen, J. G. Hernandes Cifre, and J. García de la Torre, Macromolecules 29, 3603 (1996).

${ }^{34}$ J. G. Hernandes Cifre and J. García de la Torre, J. Non-Cryst. Solids 235-237, 717 (1998).

${ }^{35}$ J. G. Hernandes Cifre and J. García de la Torre, J. Rheol. 43, 339 (1999).

${ }^{36}$ S. W. Fetsko and P. T. Cummings, J. Rheol. 39, 285 (1995).

${ }^{37}$ U. S. Agarwal, R. Bhargava, and R. A. Mashelkar, J. Chem. Phys. 108, 1610 (1998); 113, 3397 (2000).

${ }^{38}$ A. V. Lyulin, D. B. Adolf, and G. R. Davies, J. Chem. Phys. 111, 758 (1999); Macromolecules 33, 3294 (2000); 34, 3783 (2001).

${ }^{39}$ M. Fixman, J. Chem. Phys. 69, 1527 (1978); Macromolecules 14, 1710 (1981).

${ }^{40}$ D. L. Ermak and J. A. McCammon, J. Chem. Phys. 69, 1352 (1978).

${ }^{41}$ S. A. Allison and J. A. McCammon, Biopolymers 23, 167 (1984).

${ }^{42}$ R. G. Larson, Rheol. Acta 29, 371 (1990).

${ }^{43}$ P. G. De Gennes, Science 276, 5321 (1999).

${ }^{44}$ H. C. Öttinger, Phys. Rev. E 50, 2696 (1994).

${ }^{45}$ H. C. Öttinger, Stochastic Processes in Polymeric Fluids (Springer-Verlag, New York, 1996), p. 244.

${ }^{46}$ D. Petera and M. Muthukumar, J. Chem. Phys. 111, 7614 (1999).

${ }^{47}$ J. Rotne and S. Prager, J. Chem. Phys. 50, 4831 (1969).

${ }^{48}$ R. B. Bird, C. F. Curtiss, R. C. Armstrong, and O. Hassager, Dynamics of Polymeric Liquids, Vol. 2. Kinetic Theory, 2nd ed. (Wiley-Interscience, New York, 1987). 
${ }^{49}$ C. Pierleoni, G. Arialdi, and J.-P. Ryckaert, Phys. Rev. Lett. 79, 2990 (1997).

${ }^{50}$ A. Onuki, J. Phys. Soc. Jpn. 54, 3656 (1985).

${ }^{51}$ Yu. Ya. Gotlib, E. A. Karpov, and G. A. Medvedev, Polym. Sci. U.S.S.R. A31, 1043 (1989).

${ }^{52}$ T. Hoffman, R. G. Winkler, and P. Reineker, Phys. Rev. E 61, 2840 (2000).
${ }^{53}$ W. Kuhn and F. Grun, Kolloid-Z. 101, 248 (1942).

${ }^{54}$ F. S. Henyey and Y. Rabin, J. Chem. Phys. 82, 4362 (1985).

${ }^{55}$ Yu. V. Brestkin, Yu. Ya. Gotlib, and L. I. Klushin, Polym. Sci. U.S.S.R.

A31, 1143 (1989).

${ }^{56}$ O. Hassager, J. Chem. Phys. 60, 2111 (1974)

${ }^{57}$ J. M. Wiest, Polymer 40, 1917 (1999). 